\title{
ROLE OF MULTIPARAMETRIC MAGNETIC RESONANCE IN THE STUDY OF THE PROSTATE
}

Dott. TSRM Troncone Raffaella ${ }^{1}$, Dott. TSRM Coda $\mathrm{Marco}^{2}$, Dott. TSRM Aliberti Daniele 3 , Dott. Ciccone Vincenzo ${ }^{4}$, Dott. Carbone Mattia ${ }^{5}$

1 "Ospedale San Giuseppe Moscati” Taranto

${ }^{2}$ A.O.U. "San Giovanni di Dio e Ruggi d'Aragona” Salerno

${ }^{3}$ A.O.U. "San Giovanni di Dio e Ruggi d'Aragona" Salerno Dirigente Medico

${ }^{4}$ A.O.U. "San Giovanni di Dio e Ruggi d'Aragona” Salerno U.O. Radiologia Dirigente Medico

${ }^{5}$ A.O.U. "San Giovanni di Dio e Ruggi d'Aragona” Salerno Direttore f.f. U.O. Radiologia

KEYWORDS: Risonanza Magnetica Multiparametrica, 3 Tesla, Prostata, Tecnico Sanitario di Radiologia Medica, Sequenze, NEUTRA, Biopsia

\section{ABSTRACT}

Magnetic resonance imaging is the fastest and least invasive diagnostic method for the study of the prostate. The method's multiparametricity allows the acquisition of the images highlighting different characteristics in relation to the different sampling sequences.

In particular, the MRI investigation plays a role in:

- risk stratification and staging of the tumor, therefore active surveillance

- detection of prostate cancer;

- localization and characterization of prostate cancer before performing a procedure (pre-biopsy, pre-surgery, or radiation therapy);

- procedural guide (targeted biopsy guide).

The study protocol includes T2-weighted sequences on an axial and sagittal plane, diffusion-weighted sequences for the functional study (DWI) with high b values, contrast dynamic sequences (DCE-MRI), T1-weighted sequences with a large FOV.

The study protocol here presented follows the latest PI-RADS $v 2.1$ guidelines.

The axial scan plans are acquired according to the anatomical axis of the gland. In our protocol we add a further neutral T2 weighing, a sequence that will be subsequently used for the coupling of images with fusion techniques, such as target-biopsy.

The adoption of PI-RAADS v2.1, the most recent update of the system, improves the assessment of intra-patient injury variability and simplifies the general assessment of the prostate in magnetic resonance imaging.

The magnetic resonance examination thus obtained allows the correct overlap of the lesion anatomy with the ultrasound image TRUS acquired with the Fusion technique, used for targeted biopsy sampling.

\section{INTRODUCTION}

Prostate cancer is the second most common cancer and the second leading cause of death in the male population after lung cancer. [1]

The Gold Standard of investigation for prostate cancer is represented by High Field Magnetic Resonance [2] [3], which acquires a vast pattern of information and is capable of determining the malignancy of a lesion with very high sensitivity. Patients undergoing MRI investigation present with PSA elevation ( $>4 \mathrm{ng} / \mathrm{ml})$ resistant to specific therapy, with or without previous negative transrectal biopsy. The new SIU guidelines stipulate that RMmp should be performed before the biopsy.

The reference reporting system is the PI-RADS (Prostate Imaging Reporting and Data System), which defines how malignant a MRI finding is. In the short time it was published, several studies validated PI-RADS v2, but as expected, there were many ambiguities and limitations. It has therefore been updated to PI-RADS v2.1. [4]

The adoption of this updated version improves the assessment of intra-patient injury variability and simplifies the general assessment of the magnetic resonance prostate.
The study protocol presented here follows the most recent PI-RADS v2.1 guidelines.

\section{MATERIALS AND METHODS}

At the San Giovanni di Dio and Ruggi d'Aragona hospital we use Siemens Skyra multiparametric magnetic resonance equipment with 3 Tesla static magnetic field, with 16-channel Phased-Array pelvic coil. [5]

The study protocol includes T2-weighted sequences, diffusion-weighted sequences for functional study (DWI), dynamic contrast graphics sequences (DCEMRI), T1-weighted sequences with large FOV.

T2-weighted imaging gives us the opportunity to outline the zonal anatomy and to identify, locate, and stage a lesion. It also allows us to evaluate the extra-prostatic extension and the infiltration of the seminal vesicles. $\mathrm{T} 2$ weighted images are acquired in the axial plane and at least in another orthogonal plane (preferably the sagittal one). The FOV of our acquisitions is positioned orthogonally to the rectum, with coverage of the entire prostate and seminal vesicles. Sufficient coverage is obtained with 20-30 slices, 3-4 mm each and in the absence of a gap between the slices. The echo time is chosen on the basis of the intrinsic transversal relaxation values of the tissues, in order to maximize the contrast 


\begin{tabular}{|c|c|c|c|c|c|c|c|c|c|c|c|}
\hline Sequence & TE & TR & FOV & Matrix & Thickness & Distance Factor & B Value & Slices & Phase Coding & NEX & $\begin{array}{l}\text { Flip } \\
\text { Angle }\end{array}$ \\
\hline $\begin{array}{c}\text { T2W } \\
\text { Sagg. } \\
\text { Ax. } \\
\text { NEUTRAL }\end{array}$ & 100 & $\begin{array}{l}6330 \\
7500 \\
7500 \\
\end{array}$ & 200 & $320 \times 320$ & 3 & $20 \%$ & & $\begin{array}{l}22 \\
26 \\
26 \\
\end{array}$ & $\begin{array}{l}\text { H-F } \\
\text { R-L } \\
\text { R-L }\end{array}$ & 2 & 160 \\
\hline DWI & 65 & 5100 & 200 & $114 \times 114$ & 3 & $20 \%$ & $\begin{array}{c}100 \\
500 \\
1200 \\
\end{array}$ & 26 & A-P & $\begin{array}{l}2 \\
5 \\
8 \\
\end{array}$ & 160 \\
\hline DWI & 96 & 6000 & 200 & $114 \times 114$ & 3 & $20 \%$ & $\begin{array}{r}100 \\
2000 \\
\end{array}$ & 26 & A-P & $\begin{array}{l}2 \\
5 \\
\end{array}$ & 160 \\
\hline $\mathrm{T} 1 \mathrm{~W}$ & 13 & 495 & 190 & $320 \times 320$ & 5 & $10 \%$ & & 26 & R-L & 1 & 130 \\
\hline $\begin{array}{c}\text { T1 W } \\
\text { Vibe Ax. }\end{array}$ & 13 & 6000 & 380 & $320 \times 320$ & 3 & $30 \%$ & & 26 & $R-L$ & 1 & 160 \\
\hline $\begin{array}{l}\mathrm{T} 1 \mathrm{~W} \\
\text { Dixon }\end{array}$ & 1,9 & 6000 & 200 & $192 \times 192$ & 3 & $20 \%$ & & 26 & $R-L$ & 1 & 160 \\
\hline
\end{tabular}

Tab. 1 - Protocol used by our unit.

between the peripheral area (PZ) and the transition area (TZ) and between neoplastic and physiological tissue. The scanning of all sequences takes place with an acquisition plane inclined according to the prostate axis, with the exception of the sequence on the axial plane in T2 weighing called NEUTRA, in which the FOV has an inclination of 0 to allow the correct fusion of the resonance and ultrasound images using Fusion technique. [6]

Normal glandular tissue appears hyperintense in T2 weighings, and in general a lesion appears hypointense. Prostate cancer appears as a rounded hypointense focal lesion or with well-defined margins. Hypointensity, however, is not a unique sign of malignancy, in fact benign pathologies such as prostatitis, atrophy, bleeding, scars but also post-radiation alterations can lower the transversal relaxation time of the tissue and simulate a neoplasm.

Diffusion weighted imaging (DWI) are sequences designed for a functional study. Since the diffusion of water molecules is influenced by the increase in cell density and neoangiogenesis (common characteristics of a neoplasm), DWI plays the role of non-invasive marker of tumor aggression. [7] [8

The diffusion-weighted sequences are spin-eco singles-shots eco-planar, with two different values of $b$. b values greater than $800 \mathrm{~s} / \mathrm{mm}^{\wedge} 2$ stress the contrast between tissues, highlighting the signal differences of the water molecules. At lower b values there is a strong contribution from T2 weighing, therefore the displayed signal intensity reflects both the diffusion of the water and the $\mathrm{T} 2$ relaxation times of the tissues.

DWI $\mathrm{ab}>1400 \mathrm{~s} / \mathrm{mm}^{\wedge} 2$ is used to differentiate prostate cancer from benign pathologies, this is because while prostatitis and prostatic hyperplasia often show an increase in signal $a b=1000$, this signal is better suppressed at very high values of $b$ In comparison, prostate cancer, due to as its greater degree of restriction of diffusion, remains hyperintense even at very high values of b. [9] [10] From the DWI acquisitions, the ADC map (apparent diffusion coefficient) is calculated, which defines the net displacement of the water molecules in a time that reflects the diffusion gradients applied during the DWI acquisition and is not affected by a partial T2 weighting.

The measured ADC values are inversely proportional to the intensity of the b value chosen in the DWI, therefore the tumor areas have a low signal intensity on the ADC map, in contrast to their high signal intensity on native DW images. [11]

T1-weighted images are acquired using spoiled gradient-echo sequences. T1 weights are used to uniquely highlight hemorrhages, especially those post-bioptic intragiandular which appear hyperintense. The evaluation of local lymph node stations and bone metastases is also based on this weighing, with acquisition at large FOV because a lower spatial resolution represents an acceptable compromise in order to increase anatomical

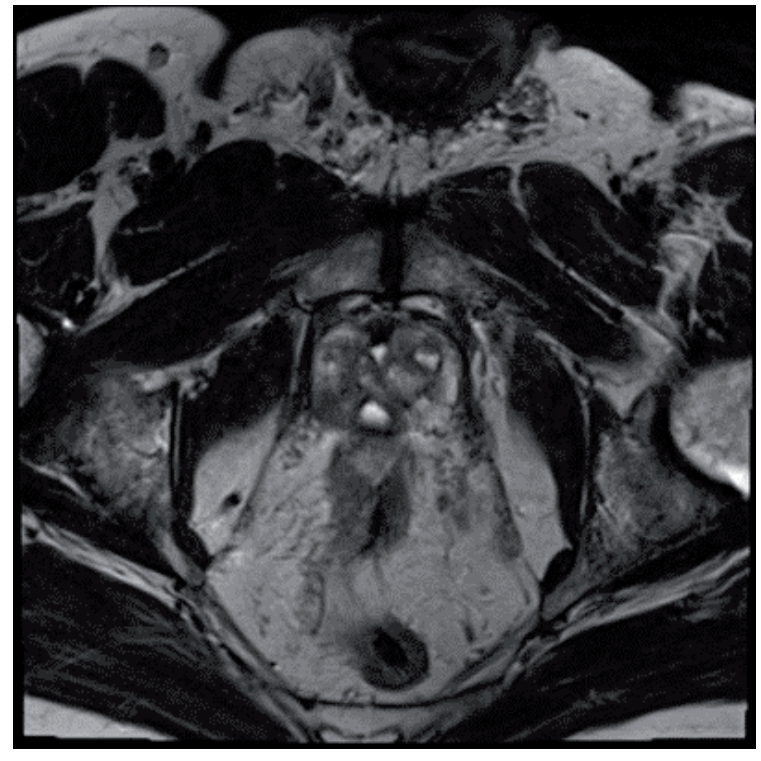

Fig. 1 - Neutral axial T2 acquisition. 

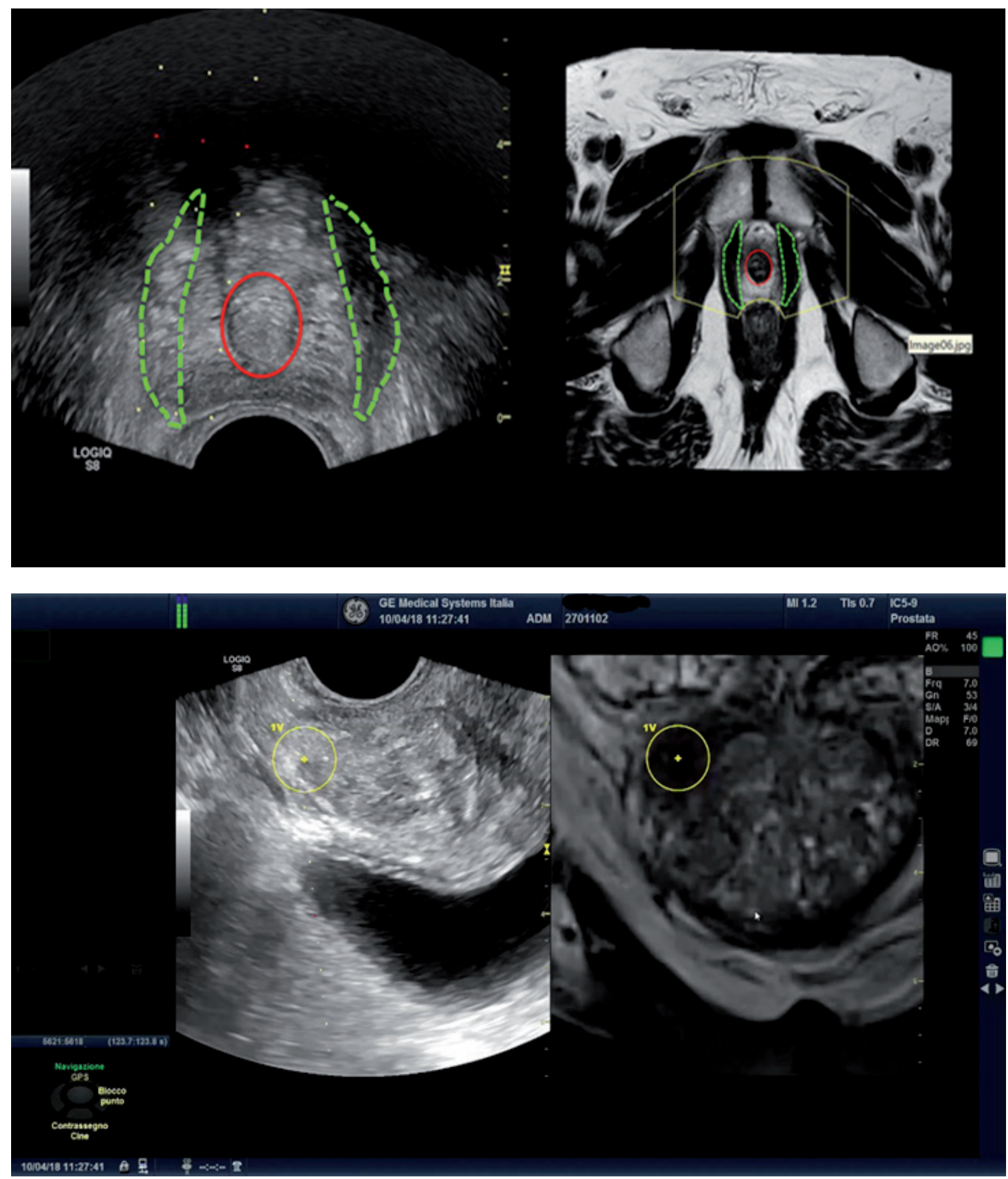

Fig. 2 e 3 - Fusion of the images of the previous MRI acquisition (right) and of the ongoing biopsy procedure.

coverage and decrease acquisition time.

The sequence of acquisition with contrast medium allows the evaluation of the vascularization and capillary permeability of a neoplasm, studying uptake and disposal of the contrast medium.

The contrast medium used is the Gadovist with a density of 0.5 administered in 10 units, sufficient for the prostate study.

After about $20 \mathrm{sec}$. from the end of the injection we proceed with the VIBE acquisition, to study an early arterial phase by defining the wash-in of the contrast. A VIBE sequence is a T1-weighted spoiled Gradient Eco designed for volumetric studies with high temporal resolution. [12]

The total duration of the exam is approximately 21 minutes.

The magnetic resonance examination thus obtained allows the correct overlap of the lesion anatomy with the TRUS ultrasound image used for targeted biopsy sampling.

For Fusion Biopsy, a V-NAV system is used, an integrated system consisting of a $3 \mathrm{D}$ ultrasound system with $7.5 \mathrm{mHz}$ END-FIRE probe and dedicated fusion software.

The advantages of the biopsy under RM-US imaging are many: this technique combines the best sensitivity of Resonance for the detection of significant tumors with the widespread availability and ease of use of TRUS. It is faster than the biopsy under direct MR guide in the gantry, and above all it does not require the use of specific non-magnetic equipment; it must not undergo the MRI machine time, in fact the systematic sampling (if desired) can be easily performed during the same biopsy session. 


\section{CONCLUSION}

Magnetic resonance imaging is the fastest and least invasive diagnostic method for the study of the prostate. The method's multiparametricity allows the acquisition of the images highlighting different characteristics in relation to the different sampling sequences. Furthermore, the acquisition of sequences of a different nature allows the intra-patient comparison of the lesion, therefore a suspected neoplasm highlighted in T2 weighing and by the functional study in DWI can be further defined as based on the characteristics highlighted by the dynamic study with contrast medium. Multiparametric Magnetic Resonance therefore represents the best method in diagnostic imaging to make the diagnoses of prostate cancer more accurate, thus improving the horizons of care for our patients.

\section{REFERENCES}

1. American Cancer Society. http//www.cancer.org/cancer/prostatercancer/detailedguide/prostate-cancer-key-statistics/.

2. Rouvière, Hartman: Prostate RM imaging at high-field strength: evolution or revolution?

3. Torricelli, Bareberini, Cinquantini: 3T MRI with phased-Array coil in local staging of prostatic cancer

4. Società italiana di Urologia (SIU) 2019 Guidelines Congresso di Riccione 2018

5. Lagemaat, Scheenen: Role of high-field MR in studies of focalized prostate cancer.

6. European Association of Urology, Turkbay, RosenKrantz, Haider... Prostate Imaging reporting and Data System Version 2.1: 2019 Update of Prostate Imaging Reporting and Data System Version 2

7. Kuhl, Gieseke, von Falkenhausen, ... Sensitivity encoding for Diffusion Weighted MR imaging at 3,0 T: intraindividual comparative study

8. Yoshizako, Wada, Hayashi, ... Usefullness of DWI and dynamic contrast enanched magnetic resonance imaging in the diagnosis of prostate transition zone cancer.

9. Katahira, Takahare,Knee, ... Ultra high b-value diffusion weighted MR imaging for the detection of prostate cancer

10. Kitajima, Kaji,Kuroda,.. High b-value diffusion weighted imaging in normal and malignant peripheral zone tissue of the prostate: effect to signal to noise ratio

11. Thormer, Otto, Reiss Zimmermann, ... Diagnostic value of ADC in patience with prostate cancer: influence of the choice of $b$ value.

12. Panebianco, Barchetti, Sciarra: Prostate cancer recurrence after radical prostatectomy: the role of 3 Tesla diffusion imaging in multi-parametric magnetic resonance imaging. 